\title{
ACCELERATING TWO-SCALE SIMULATIONS WITH A NON-INTRUSIVE REDUCED ORDER MODEL
}

\author{
Theron Guo $^{1 *}{ }^{*}$ Karen Veroy ${ }^{1}$ \\ ${ }^{1}$ Centre for Analysis, Scientific Computing and Applications \\ Department for Mathematics and Computer Science \\ Eindhoven University of Technology \\ *E-mail: t.guo@tue.nl
}

In order to understand the structure-property relations of complex materials, two-scale material simulations utilizing computational homogenization can be used. In computational homogenization the microstructure is explicitly defined on a so-called representative volume element, hence replacing the macroscopic constitutive model and yielding a coupled formulation which can be solved with a multilevel finite element scheme. Due to high computational expenses, such simulations can be infeasible in multi-query contexts such as optimization, control or design. To make such analyses possible, in this work, a surrogate model for the microscopic simulation based on reduced basis methods [1] is proposed. After a few high-fidelity simulations have been computed and collected, the surrogate is constructed in two steps. In the first step, a proper orthogonal decomposition is applied on the stress field to extract a global lowdimensional basis that can sufficiently approximate the stress. Then, a regression based on deep neural networks $[2,3]$ is employed to find the basis coefficients for any parameter. Given any arbitrary new parameter, the microscopic field is rapidly obtained, from which the effective stress and stiffness can be analytically derived. Furthermore, the derivatives of the stress with respect to material parameters are readily available and can thus be used for optimization or design. To illustrate the accuracy of the surrogate model, a two-scale problem involving a fiber-reinforced composite microstructure is presented. The macroscopic solution obtained with the surrogate model is compared to the full two-scale simulation. The results showcase the high accuracy of the proposed method on both macro- and microscale despite using a relatively small training dataset and highlight the high efficiency of the method.

Acknowledgement: This result is part of a project that has received funding from the European Research Council (ERC) under the European Union's Horizon 2020 research and innovation programme (Grant agreement No. 818473).

\section{References}

[1] Quarteroni, A., Manzoni, A. and Negri, F., 2015. Reduced basis methods for partial differential equations: an introduction (Vol. 92). Springer.

[2] Hesthaven, J.S. and Ubbiali, S., 2018. Non-intrusive reduced order modeling of nonlinear problems using neural networks. Journal of Computational Physics, 363, pp.55-78.

[3] Mohan, A.T. and Gaitonde, D.V., 2018. A deep learning based approach to reduced order modeling for turbulent flow control using LSTM neural networks. arXiv preprint arXiv:1804.09269. 